\title{
An In-Vivo Study during Combined Intracavitary and Interstitial Brachytherapy of Gynaecological Malignancies Using microMOSFET*
}

\section{Ramapandian Seenisamy ${ }^{1}$, Vivekanandan Nagarajan ${ }^{2}$, Ashutosh Mukherji1, Parthasarathy Vedasoundaram ${ }^{1}$, K. S. Reddy ${ }^{3}$, Vivekanandam Singhavajala ${ }^{1}$, Vijayaprabhu Neelakandan ${ }^{1}$}

${ }^{1}$ Department of Radiotherapy, RCC, JIPMER, Puducherry, India

${ }^{2}$ Department of Medical Physics, Cancer Institute, Adyar, Chennai, India

${ }^{3}$ Department of Oncology, MGMC \& RI, Puducherry, India

Email: nellaiseeni@gmail.com

How to cite this paper: Seenisamy, R., Nagarajan, V., Mukherji, A., Vedasoundaram, P., Reddy, K.S., Singhavajala, V. and Neelakandan, V. (2017) An In-Vivo Study during Combined Intracavitary and Interstitial Brachytherapy of Gynaecological Malignancies Using microMOSFET. International Journal of Medical Physics, Clinical Engineering and Radiation Oncology, 6, 162-173. https://doi.org/10.4236/ijmpcero.2017.62015

Received: April 11, 2017

Accepted: May 20, 2017

Published: May 23, 2017

Copyright (c) 2017 by authors and Scientific Research Publishing Inc. This work is licensed under the Creative Commons Attribution International License (CC BY 4.0).

http://creativecommons.org/licenses/by/4.0/

\begin{abstract}
Aim: To analyze the inter-fraction, intra-fraction uncertainties and to verify the delivered total dose with planned dose in the combined intracavitary-interstitial brachytherapy of gynaecological cancer patients using microMOSFET in-vivo dosimeter. Materials and Methods: Between May 2014 and March 2016, 22 patients who underwent brachytherapy treatments with an applicator combination of CT/MR compatible tandem, ring and Syed-Neblett template-guided rigid needles were included in this study. Specially designed microMOSFET, after calibration, was used to analyze the variations in dosimetry of combined intracavitary-interstitial application. Results: The standard deviation for Inter-fraction variation among 22 combined intracavitary interstitial applications ranged between $0.86 \%$ and $10.92 \%$. When compared with the first fraction dose, the minimum and maximum dose variations were $-9.5 \%$ and $26.36 \%$, respectively. However, the mean doses varied between $-5.95 \%$ and $14.49 \%$. Intra-fraction variation, which is the difference of TPS calculated dose with first fraction microMOSFET-measured dose ranges from $-6.77 \%$ to $8.68 \%$. The variations in the delivered total mean dose in 66 sessions with planned doses were $-3.09 \%$ to $10.83 \%$. Conclusions: It is found that there was a gradual increase in microMOSFET measured doses as compared to the first fraction with that of subsequent fractions in 19 out of 22 applications. Tumor deformation and edema may be the influencing factors, but the applicator movements played a major role for the variations. We find that
\end{abstract}


the microMOSFET is an easy and reliable system for independent verification of uncertainties during ICBT-ISBT treatments.

\section{Keywords}

microMOSFET, Intra-Fraction Variations in Brachytherapy, Inter-Fraction Variations in Brachytherapy, Combined Intracavitary and Interstitial Brachytherapy, In-Vivo Dosimetry in Brachytherapy, Applicator Displacement, Brachytherapy Uncertainties

\section{Introduction}

Brachytherapy is used to deliver high doses limited to the accessible tumors, with minimal doses in the surrounding normal tissue regions. Though Intracavitary Brachytherapy (ICBT) is generally used in most of the gynaecological tumors, some patients with paravaginal and parametrial involvement, and with bulky and complex shaped tumour require Interstitial Brachytherapy (ISBT) as well [1]. ICBT + ISBT delivers better CTV coverage compared to the suboptimal doses from ISBT alone [2]. Syed-Neblett Template (SNT), Martinez Universal Perineal Interstitial Template (MUPIT) and Vienna applicator are used as implant templates for such treatments.

The delivered dose has to be checked and verified with planned dose since precise and accurate dose delivery is essential in radiotherapy. In-vivo dosimeters like TLD, MOSFET, diodes, diamond detector and scintillation detector are used for dose delivery verifications in brachytherapy [3].

Metal Oxide Semiconductor Field Effective Transistor (MOSFET) is a wellaccepted in-vivo dosimeter due to its simple design, user-friendly functionality and independence of dose rate, radiation energy, beam angular characters and real-time measurements as no post-irradiation processing required [4] [5]. The shift in threshold voltage of MOSFET before and after treatment is directly proportional to absorbed dose. Specially-designed microMOSFET is used for brachytherapy due to their smaller size that can be inserted inside the rigid needles as well as $6 \mathrm{~F}$ flexible tubes. MOSFETs need to be calibrated with concern radiation generating source before their clinical use.

During multisession brachytherapy, there is a likelihood of changes in the position and orientation of the catheters [6] [7]. Reimaging and re-planning may reduce these inter-fractional variations to certain extent, but intra-fractional uncertainty still remains. Errors in applicator reconstruction; source positioning and identification of the applicator during treatment translate into the changes in delivered doses. Moreover, the contouring and planning take about $3-4$ hours between imaging and delivery, thereby increasing the chances of displacement of applicators positions and organ motion. Due to sharp dose gradients in Brachytherapy makes such changes in applicator positions and organ motions result in gross errors in dose delivery. If any error is found during the 
first fraction, intervention before subsequent treatment sessions is possible, and the action level may be chosen to stop unintended doses. These issues demand the dose verification system for each treatment plan.

In this study, we have analyzed the inter-fraction and intra-fraction variations in the treatment of combined ICBT + ISBT using the microMOSFET in-vivo dosimeter. We compared the measured point dose during the first fractionation session with that of the TPS calculated point dose to study intra-fraction variations. For inter-fraction variations, delivered doses in subsequent sessions are compared with the dose measured during the first fraction.

\section{Materials and Methods}

Between May 2014 and March 2016, a total of 66 fractionation sessions of 22 patients with combined CT/MR compatible applicator consisting of tandem and ring with Syed-Neblett Template-guided 8 - 10 rigid needles were included for this study (Figure 1). CT imaging was done on the same day, within 4 hours from the application, to minimize the treatment duration. Dual-slice Somatom Spirit Power CT-simulator (M/s Siemens Medical Solutions, Germany) was used for imaging of all patients. 3D brachytherapy planning was done using Varian Brachyvision ver.10.0.0 (M/s Varian Medical Systems, UK) Treatment Planning System (TPS) that uses TG-43 formalism for dose calculations. Prescribed dose for the combined applicator was 15 Gy in 3 fractions normalized to Point A and ensuring adequate dose coverage to the CTV. Treatments were delivered using GammaMed Plus iX High Dose Rate unit (M/s Varian Medical Systems, UK) with Ir-192 single stepping source. Specially-designed microMOSFET (M/s Best ${ }^{\circledR}$ Medical, Canada) was used as an in-vivo dosimeter.

\subsection{Combined Applicator}

Combined Intracavitary and Interstitial applicators, i.e., ICBT + ISBT is used for locally-advanced, irregularly-shaped, large-volume tumors with pelvic and vaginal involvements. ICBT + ISBT offers a flexible way of customized treatment for each patient. Position and number of the needles in template depend on the tumor size and location of each patient [8]. Needles were inserted to a pre-determined pattern to cover the CTV. Tightening the screws keeps the needles in position relative to the perineal plate and sutures were put to maintain the plates in

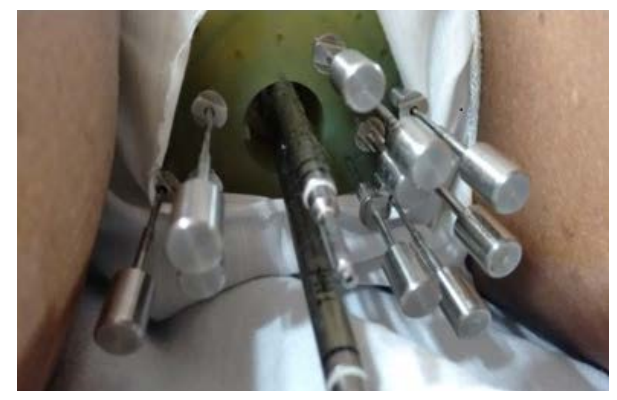

Figure 1. Combined applicator of Tandem, Ring and Syed-Neblett Template with rigid needles. 
their original position. The central cavity in the template allowed for the application of a uterine tandem and ring intracavitary implant for the dose to the uterine fundus and cervix. The template was tied to the pelvis of the patient to arrest the movement, and external wrapping of needles with gauze piece was done to minimize single needle movement during in-patient care.

\subsection{Treatment Planning}

CT imaging with $3 \mathrm{~mm}$ slice thickness was obtained for all patients. The CTV, bladder, and rectum were contoured as per the GEC-ESTRO guidelines. TG-43 formalism was used for calculation of all treatment plans. Though geometric and volumetric optimization methods are available, we preferred the equal dwell time technique and further improvement made by small adjustments in dwell times manually. Prescribed dose for ICBT + ISBT was $15 \mathrm{~Gy}$ in 3 fractions normalized at Point A. Plan evaluations and approvals were done as per GECESTRO guidelines.

\subsection{Treatment Delivery}

Approved plans were imported from TPS to the control console system of HDR through DICOMRT. Labeling of applicators was done as per plan. A gentle press was applied without disturbing catheters' position while connecting and disconnecting the guide tube. Before each fraction, physical verification of needle's position was also done. All patients were treated with Gamma MedPlus iX HDR unit.

\section{4. microMOSFET}

Commercially-available microMOSFET in-vivo dosimeter (Standard) Model TN-502RDM (M/s Best ${ }^{\oplus}$ Medical Canada) was used in this study. Since the study was done with 5 Gy dose, we used the standard bias settings. This setting enhances the lifetime of the microMOSFET [9]. The microMOSFET metal leads have special $2.5 \mathrm{~mm}$ graduated marks in brown and white alternately, to allow for easy measurement of the detector position inside the catheter and consistent positioning for multisession studies. The advantage of microMOSFET over the standard MOSFET is the minimal angular dependence, $\pm 1 \%[10]$.

\subsection{Calibration of microMOSFET}

Five microMOSFET probes were used in this study and calibrated independently in the Ir-192 source with known calculated dose before their usages. Varianmade catheter flap applicator with the rigid needles was used for the calibration of microMOSFET with Ir-192 HDR source. Clinically-relevant distance in Brachytherapy is $1 \mathrm{~cm}$ to $5 \mathrm{~cm}$, for which the accuracy of the measurements for MOSFETs lie within $\pm 7 \%$ for Ir-192 [11]. Instead of calibrating microMOSFET at different distances, we tried to get the collective readings of the different needles at a different distance. Equally-spaced 11 catheters with $1-\mathrm{cm}$ inter-catheter separations were used for this calibration procedure (Figure 2). CT image 


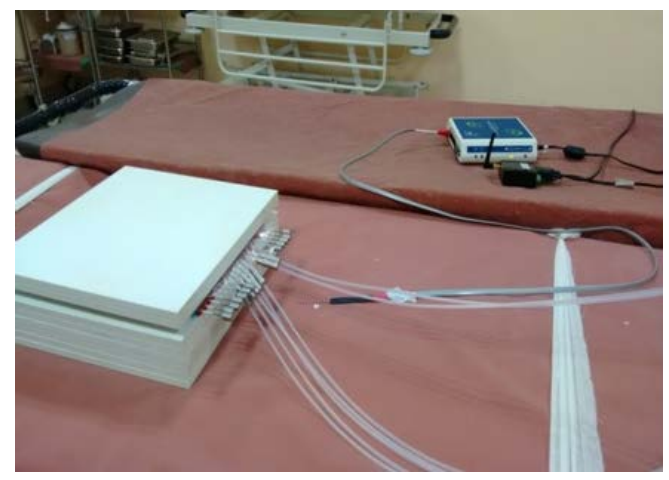

Figure 2. Calibration of microMOSFET with Iridium-192 in rigid needle setup.

of the applicator with eleven catheters was taken, and a 3D plan was done for 100 cGy normalized at microMOSFET's position with equal dwell times. Treatment length of each needle was $5 \mathrm{~cm}$ with the first dwell position at $5 \mathrm{~cm}$ from the tip, and each microMOSFET was placed at $7.5 \mathrm{~cm}$ from the tip of the middle, i.e., the $6^{\text {th }}$ catheter. Calibration was repeated for a total of fifteen times in five days for five microMOSFETs independently, and the average was taken as a calibration factor for the Ir-192 source in rigid needle applications. The linearity of the microMOSFET probes were also checked with doses varying from $10 \mathrm{cGy}, 20$ cGy, 50 cGy, 1 Gy, 2 Gy, 3 Gy and 5 Gy. Dose-rate dependency was checked with different activities of the Ir-192 sources at various points of time.

\subsection{Inter-Fraction Variations Study of Combined Applicator}

The last catheter of each implant was used for the placement of microMOSFET. The position of the microMOSFET was in the centre of the active length of the last catheter, which depends on each patient's CTV. Distance from the tip of the applicator was $2-5 \mathrm{~cm}$ for microMOSFET placement for different patients, and the same is maintained with the colored marking as well as physically checked with a ruler. The first fraction position, where the microMOSFET was just exiting from the catheter proximal position, was marked and the same position of the probe was reproduced for the remaining fractions. Distal blind end of the microMOSFET metal lead was pasted with micropore tape to the entry of catheter to fix the position during the measurements. Before starting the treatment microMOSFET threshold values were noted. First fraction reading was taken as the baseline value, and the subsequent measurements for the particular plan were compared with that for inter-fraction variations.

\subsection{Intra-Fraction Variations Study of Combined Applicator}

For this study, additional 22 plans were generated, which were copies of the treatment-approved plans, but without source dwell positions in the last catheters. The last catheter was used for the placement of microMOSFET probe and calculations were done keeping the remaining dwell positions and their dwell times same. A new reference point was created for each plan which exactly matching with the microMOSFET position in the delivered plan. These TPS- 
calculated point doses were noted and compared with the microMOSFET measured point doses during the first fraction of each patient. This intra-fraction study eliminates gross errors during Brachytherapy.

\subsection{Verification of the Delivered Total Dose with Planned Dose}

This method is similar to that intra-fraction study, but the total mean measured doses noted during three fractions for each patient is compared with that of calculated dose at the microMOSFET's position.

\section{Results}

We included 22 combined applications of ICBT + ISBT for this study. All patients have completed their treatment course as per plan without interruptions or complications. The calibration factor of micro-MOSFETs with Ir-192 in rigid needle applicator was calculated to be $0.9372 \mathrm{cGy} / \mathrm{mV}$.

The inter-fraction variations of delivered doses for the combined ICBT + ISBT of all patients are shown in Figure 3, keeping the first fraction dose as $100 \%$. Percentage standard deviation (SD) of inter-fraction variations ranges from $0.86 \%$ to $10.92 \%$ (Figure 4 ). The minimum and maximum values of inter -fraction variations were $-9.5 \%$ and $26.36 \%$, respectively (Figure 5).

Intra-fraction variations or the deviations in delivered first session doses with that of calculated point doses were ranged from $-6.77 \%$ to $8.68 \%$ (Figure 6). But in 19 applications, the deviation was within $\pm 5 \%$.

The results of the study for verifying the delivered mean dose of all sessions with that of the planned dose is shown in Figure 7.

TPS calculated point doses, TG-43, microMOSFET measured first fraction doses and delivered mean doses of all patients in cGy were shown in Table 1.

\section{Discussion}

In this study, we found that the dose measured was found increasing in successive fractions. The CT imaging was done within 4 hours and the

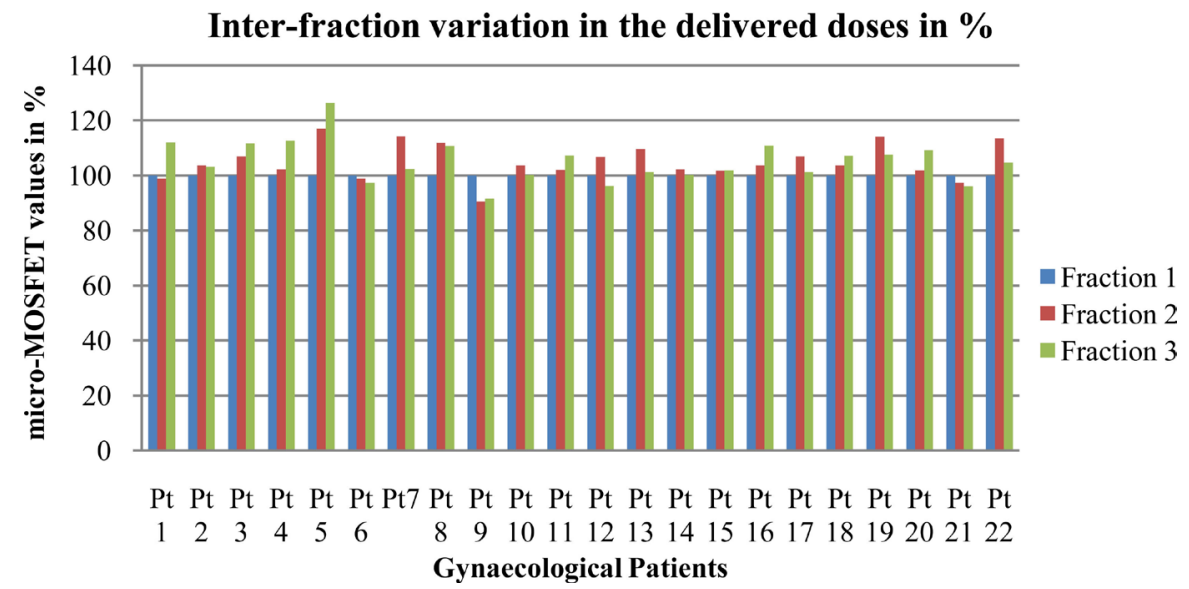

Figure 3. Inter-fraction variations in the delivered doses of multi-fractionated gynaecological patients in percentage. 


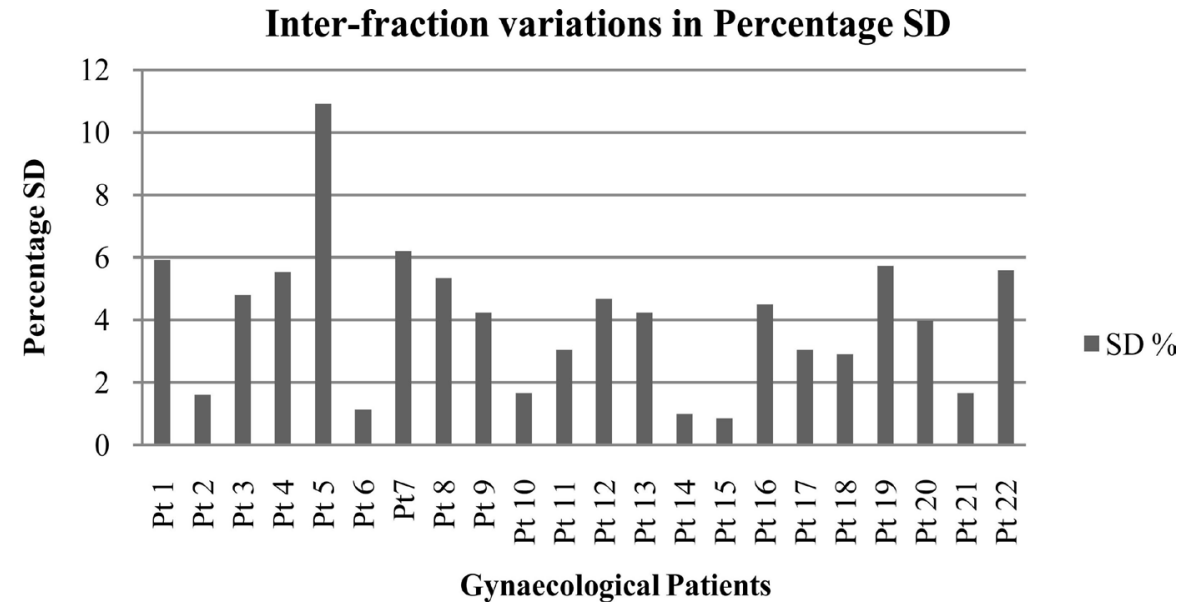

Figure 4. Inter-fraction Variations in the delivered doses of multi-fractionated gynaecological patients in Percent Standard Deviation.

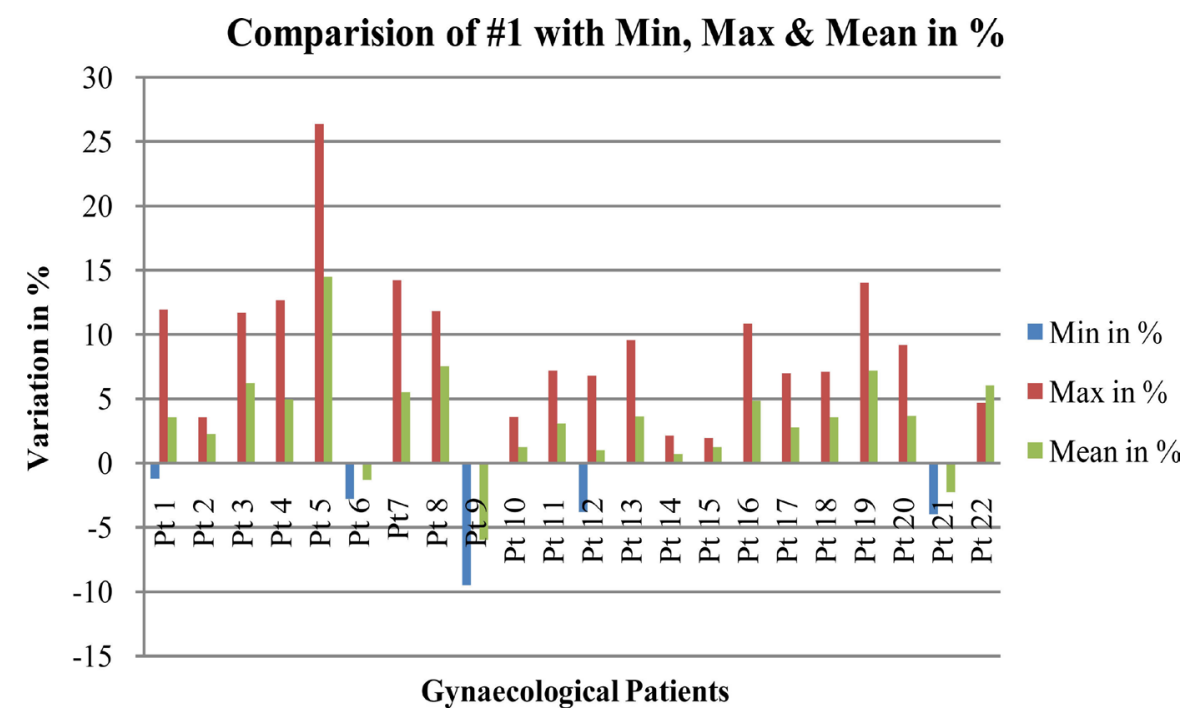

Figure 5. Comparison of fraction 1 doses with minimum, maximum and mean doses in percentage.

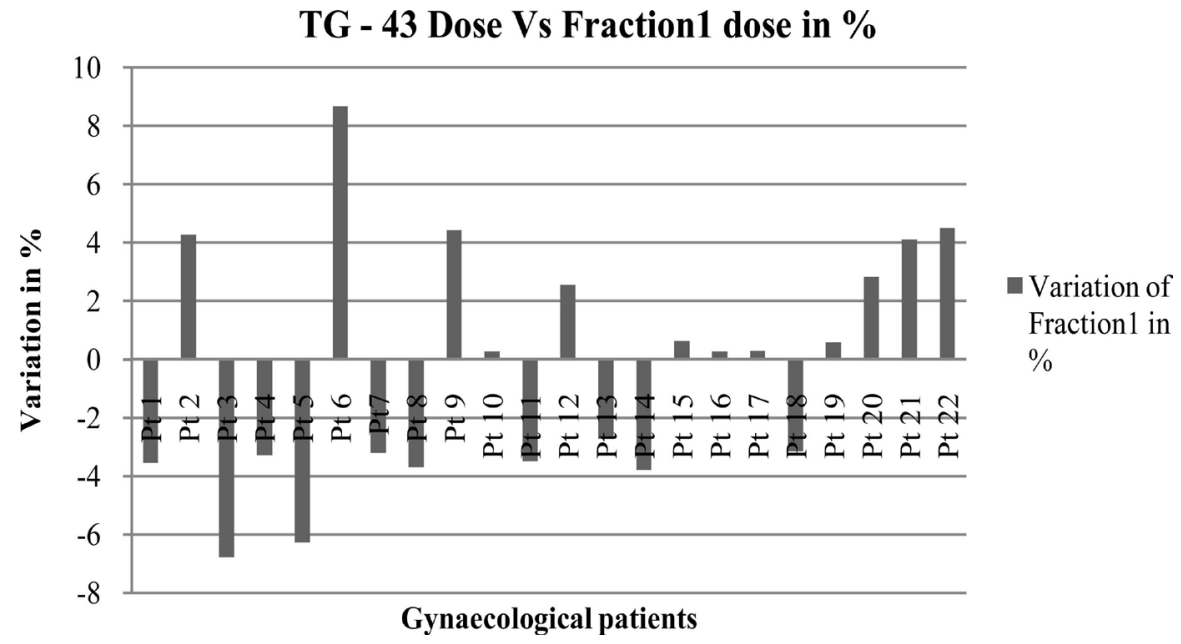

Figure 6. Comparison of TPS calculated, TG-43, point doses with fraction 1 doses in percentage. 
TG-43 Dose Vs Mean dose in \%

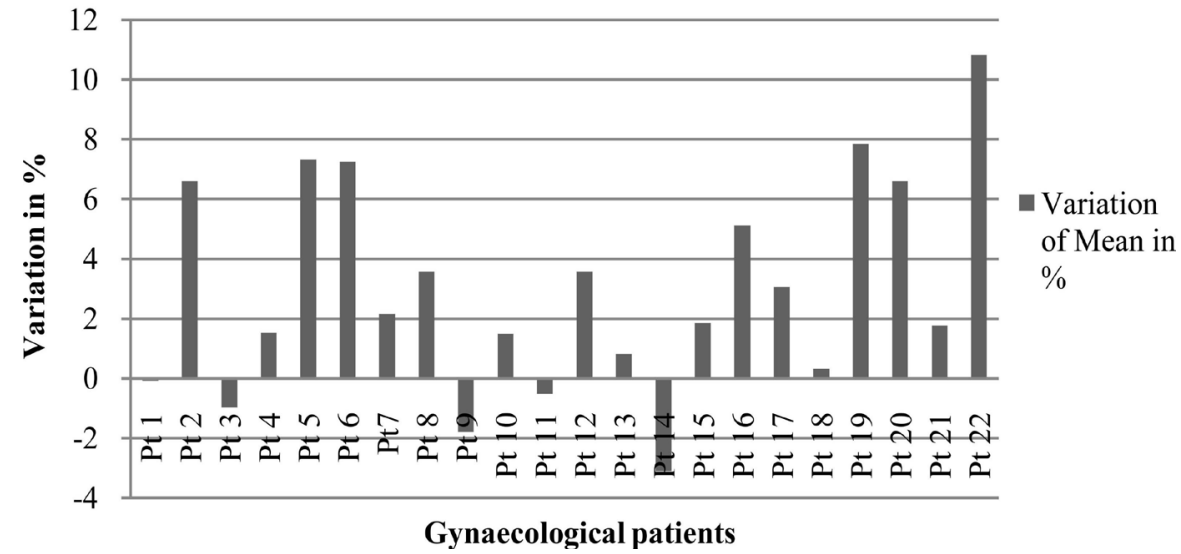

Figure 7. Comparison of TPS calculated, TG-43, point doses with mean doses in percentage.

Table 1. TPS calculated, TG-43, point doses, first fraction doses and mean delivered doses of all patients in cGy.

\begin{tabular}{|c|c|c|c|}
\hline \multirow{2}{*}{ Sr. No. } & \multicolumn{3}{|c|}{ Point doses in cGy } \\
\hline & TPS calculated & First fraction & Mean \\
\hline Pt 1 & 162.5 & 156.75 & 162.36 \\
\hline Pt 2 & 740.7 & 772.26 & 789.59 \\
\hline Pt 3 & 915 & 853.09 & 906.19 \\
\hline Pt 4 & 598.2 & 578.6 & 607.26 \\
\hline Pt 5 & 710 & 665.54 & 761.92 \\
\hline Pt 6 & 316 & 343.41 & 338.86 \\
\hline Pt7 & 285 & 275.89 & 291.14 \\
\hline Pt 8 & 195 & 187.8 & 201.95 \\
\hline Pt 9 & 558.2 & 582.89 & 548.21 \\
\hline Pt 10 & 272.9 & 273.61 & 276.96 \\
\hline Pt 11 & 160.5 & 154.92 & 159.68 \\
\hline Pt 12 & 246 & 252.27 & 254.77 \\
\hline Pt 13 & 313 & 304.49 & 315.54 \\
\hline Pt 14 & 637.2 & 613.07 & 617.53 \\
\hline Pt 15 & 896.2 & 901.84 & 912.76 \\
\hline Pt 16 & 620 & 621.65 & 651.7 \\
\hline Pt 17 & 850 & 852.4 & 875.9 \\
\hline Pt 18 & 301.5 & 292.01 & 302.46 \\
\hline Pt 19 & 510 & 513 & 549.96 \\
\hline Pt 20 & 665.54 & 684.28 & 709.44 \\
\hline Pt 21 & 315 & 327.92 & 320.56 \\
\hline Pt 22 & 295 & 308.27 & 326.92 \\
\hline
\end{tabular}


treatments were commenced on the same day of applications to minimize the treatment durations and to reduce the complications due to the implanted needles in the pelvic region. Consequently, the variation in dose measurements owing to the healing of tissue oedema is substantial during first and subsequent fractions. Onoe et al. [6] reported that oedema in perineal area pushes the applicators towards the caudal direction. This results in moving the measuring point close to the ring applicator in most of the incidences. They found the solution to overcome the cranio-caudal displacement of needles with the help of fully stretched elastic tape. They pasted the tape with the template assembly on the patient. This method drastically cut down the D90 of CTV within $\pm 5 \%$ in contrast with their earlier study of $35 \%$ to $40 \%$. We have used gauze piece to tie down the template with the patient apart from the suture. This method also helped us to keep the mean delivered dose within $\pm 10 \%$.

Based on planning study, Rey et al. [7] reported that CTV dose coverage decreased in day 3 and suggested for re-planning on day two before the second or third fractions. In our in-vivo dosimetry study, we found that there was a gradual increase in the delivered doses in 17 patients, which could be because of tumor shrinkage during the treatment process itself. Tumor shrinkage brings the implanted needles in them to move closer to each other and would proportionally increase the dose to the measuring positions. Other reasons could be (i) because a gentle press had to be applied while connecting and disconnecting the guide tube from HDR to the needle; (ii) shifting of patients up to the treatment room, both would result in deforming the original applicator geometry. Needle movement could bring microMOSFET position close to the ring applicator hence the delivered dose was increased for most of the patients. Applicator movement of $\pm 3 \mathrm{~mm}$ caused a dosimetric change of greater than $10 \%$ for ICBT (Schindel et al. [12]). As we observed that the measured mean delivered dose variations ranged from $-3 \%$ to $8 \%$, the needle displacements in this study could be less than $\pm 3 \mathrm{~mm}$.

Khinikar et al. [5] found the angular dependence is $\pm 6 \%$ for standard MOSFET with Ir-192, but Chung et al. [10] found the angular dependence of microMOSFET was $\pm 1 \%$ in the electron beam. The rigid metal needles used in this study are almost straight. Therefore, error in the reproducibility of microMOSFET position is negligible for the entire treatment and direction facing the radiation beam can be maintained.

The sensitivity of microMOSFET with distance from the Ir-192 was investigated by Qi et al. [11]. Their result showed that the variations were within $\pm 7 \%$ for the distance of $1-5 \mathrm{~cm}$. Since the length of the needle and tandem is large, small displacements could further enhance the variation in the sensitivity of microMOSFET. To overcome this, the microMOSFET was placed at the centre of the active length of that needle. This method effectively reduced the distance of all dwell positions to $1-5 \mathrm{~cm}$ from microMOSFET position.

One of the ways to minimize the inter-fraction variation is to repeat CT imaging before each fraction (Velmurugan et al. [13]). Kandasamy et al. [14] con- 
cluded that repeat-CT on the fifth day from the application is sufficient. CT/MRI imaging before each fraction can be an effective way to find the tumor shrinkage, edema and applicator displacement. However, the adaptive planning estimates the dose according to imaging position only, but the delivered dose is more of manipulation than reality. We found the superiority of microMOSFET in-vivo dosimetry method over CT dosimetry method in that the verification of dose delivered is done during the treatment process. The combination of both methods shall cut down the error before as well as during treatment.

We studied inter-fraction variations by direct measurement of the dose delivered at a point inside the CTV using microMOSFET in-vivo dosimeter. The measured dose during the first fraction was taken as the baseline value and compared it with all subsequent ICBT + ISBT sessions (Figure 5). In 17 patients, the measured value during first fraction was found to be minimum compared to subsequent fractions. Thus, there is an increase in the dose at the point of dose measurement, which could be due to tumor shrinkage and edema resolution and thus, needles could have migrated close to each other. However, in the remaining five patients, the minimum deviation was $-9.5 \%$ found in the subsequent fractions, and the maximum dose was measured in the first fractions in three of these five patients. This could be due to the displacements of the applicator as well as microMOSFET positions. Largest maximum dose deviation was $+26.36 \%$ when all patients were included, but it is less than $14 \%$ among 18 applications. The mean values of inter-fraction deviations among 22 patients ranged from $-5.95 \%$ to $7.54 \%$ except for one patient, where the value was $+14.49 \%$. Thus, in the majority of the patients, the inter-fraction variations were well below $\pm 10 \%$ during the combined ICBT + ISBT applications.

In the intra-fraction variation study, TPS point dose calculated at the microMOSFET position was taken as a baseline value for all 22 plans and were compared with their respective first fraction measured values. Of the 22 values, 10 dose values fall on the negative side with variations ranging from $-6.77 \%$ to $-2.72 \%$. In the remaining 12 patients, the intra-fraction variations were in the range of +0.27 to +8.68 . Thus, the intra-fraction variation, which is the difference in the TPS-calculated point dose to the measured dose, the measured doses were almost equally distributed as either less or more than the calculated dose among the patients studied and the variations were well within $\pm 10 \%$ (Table 1).

During the verification of the delivered total dose with that of the planned dose, where the TPS-calculated point dose for a fraction in each patient was compared with the mean value of measured doses at the same point in all three fractions for the patient. The deviations ranged from $-3.09 \%$ to $+7.84 \%$ among 21 applications and in one case it was found to be $+10.83 \%$ (Figure 7 ). The deviations with negative values, where the measured mean values were less than calculated value, ranged from $-0.052 \%$ to $-3.09 \%$, with an average of $-1.19 \%$, were noted in 5 patients. The positive values in the deviations, when the measured values were more than calculated values ranged from $+0.32 \%$ to $+10.83 \%$ with an average of $+4.22 \%$ among the remaining 17 patients. The deviation of 
the mean measured value with that of calculated value was more than $10 \%$ was noted in one patient, though the measurement of the first fraction deviated from calculated value only by $+4.50 \%$ (Table 1 ). The deviations in the second and third fractions for this patient were $+18.57 \%$ and $+9.38 \%$, respectively. The variations were attributable to the physical displacement of this needle towards high-dose gradient region during the second and third fractions. Overall, the deviations in all patients except one patient were less than $\pm 10 \%$, and thus, during the combined ICBT + ISBT applications the doses were delivered as per the planning within acceptable deviations.

\section{Conclusions}

In this study, we found that there was a gradual increase in the inter-fraction variations, i.e., when the first fraction microMOSFET-measured delivered doses were compared with that measured doses of subsequent fractions in 19 out of 22 applications. Tumor shrinkage and edema resolutions may be the influencing factors, but applicator movements play a major role for the observed variations. As for intra-fraction variations, which are the dose difference between the calculated doses with that measured doses during the first fraction of 22 patients, it is found in this study that the deviations were within $\pm 10 \%$. When the calculated doses were compared with the mean measured doses, it is found that the variations in the dose delivery were also within $\pm 10 \%$, except for one patient.

Based on this study, we find that in-vivo dosimetry needs to be done during HDR Brachytherapy as well to monitor the dose delivery uncertainties and to stop the gross errors. An action level to intervene the treatment may be fixed as $\pm 10 \%$ for intra-fraction, inter-fraction, and dose delivery uncertainties. We find the microMOSFET is an easy and reliable system for independent in-vivo dosimeter during ICBT + ISBT treatments for monitoring inter-fraction and, intrafraction uncertainties and for delivered dose verifications.

\section{References}

[1] Liu, Z., Guo, J., Lin, X., Wang, H., Qiu, L., Ren, X., et al. (2016) Clinical Feasibility of Interstitial Brachytherapy Using a "Hybrid" Applicator Combining Uterine Tandem and Interstitial Metal Needles Based on CT for Locally Advanced Cervical Can-cer. Brachytherapy, 15, 562-569. https://doi.org/10.1016/j.brachy.2016.06.004

[2] Oike, T., Ohno, T., Noda, S.E., Kiyohara, H., Ando, K., Shibuya, K., et al. (2014) Can Combined Intracavitary/Interstitial Approach Be an Alternative to Interstitial Brachytherapy with the Martinez Universal Perineal Interstitial Template (MUPIT) in Computed Tomography-Guided Adaptive Brachytherapy for Bulky and/or Irregularly Shaped Gynecological Tumors? Radiation Oncology, 9, 222. https://doi.org/10.1186/s13014-014-0222-6

[3] Lambert, J., Nakano, T., Law, S., Elsey, J., McKenzie, D.R. and Suchowerska, N. (2007) In Vivo Dosimeters for HDR Brachytherapy: A Comparison of a Diamond Detector, MOSFET, TLD, and Scintillation Detector. Medical Physics, 34, 17591765. https://doi.org/10.1118/1.2727248

[4] Ramaseshan, R., Kohli, K.S., Zhang, T.J., Lam, T., Norlinger, B., Hallil, A., et al. 
(2004) Performance Characteristics of a MicroMOSFET as an in Vivo Dosimeter in Radiation Therapy. Physics in Medicine and Biology, 49, 4031. https://doi.org/10.1088/0031-9155/49/17/014

[5] Kinhikar, R.A., Sharma, P.K., Tambe, C.M. and Deshpande, D.D. (2006) Dosimetric Evaluation of a New OneDose MOSFET for Ir-192 Energy. Physics in Medicine and Biology, 51, 1261. https://doi.org/10.1088/0031-9155/51/5/015

[6] Onoe, T., Nose, T., Yamashita, H., Yoshioka, M., Toshiyasu, T., Kozuka, T., et al. (2013) High-Dose-Rate Interstitial Brachytherapy for Gynecologic MalignanciesDosimetric Changes during Treatment Period. Journal of Radiation Research, 54, 663-670. https://doi.org/10.1093/jrr/rrs130

[7] Rey, F., Chang, C., Mesina, C., Dixit, N., Teo, B.K. and Lin, L.L. (2013) Dosimetric Impact of Interfraction Catheter Movement and Organ Motion on MRI/CT Guided HDR Interstitial Brachytherapy for Gynecologic Cancer. Radiotherapy Oncology, 107, 112-116. https://doi.org/10.1016/j.radonc.2012.12.013

[8] Syed, A.N., Puthawala, A., Fleming, P., Vora, N., Neblett, D.L., George, F.W., et al. (1979) Interstitial-Intracavitary (Syed-Neblett) Applicator in the Treatment of Carcinoma of Cervix. International Journal of Radiation Oncology Biology Physics, 5, 204. https://doi.org/10.1016/0360-3016(79)90523-6

[9] Soubra, M., Cygler, J. and Mackay, G. (1994) Evaluation of a Dual Bias Dual Metal Oxide-Silicon Semiconductor Field Effect Transistor Detector as Radiation Dosimeter. Medical Physics, 21, 567-572. https://doi.org/10.1118/1.597314

[10] Chung, J.B., Lee, J.W., Suh, T.S., Lee, D.H., Choe, B.Y., Kim, Y.S., et al. (2009) Dosimetric Characteristics of Standard and Micro MOSFET Dosimeters as in-Vivo Dosimeter for Clinical Electron Beam. Journal of the Korean Physical Society, 55, 2566-2570. https://doi.org/10.3938/jkps.55.2566

[11] Qi, Z.Y., Deng, X.W., Cao, X.P., Huang, S.M., Lerch, M. and Rosenfeld, A. (2012) A Real-Time in Vivo Dosimetric Verification Method for High-Dose Rate Intracavitary Brachytherapy of Nasopharyngeal Carcinoma. Medical Physics, 39, 6757-6763. https://doi.org/10.1118/1.4758067

[12] Schindel, J., Zhang, W., Bhatia, S.K., Sun, W. and Kim, Y. (2013) Dosimetric Impacts of Applicator Displacements and Applicator Reconstruction-Uncertainties on 3D Image-Guided Brachytherapy for Cervical Cancer. Journal of Contemporary Brachytherapy, 5, 250. https://doi.org/10.5114/jcb.2013.39453

[13] Velmurugan, T., Sukumar, P., Krishnappan, C. and Boopathy, R. (2010) Study of Dosimetric Variation due to Interfraction Organ Movement in High Dose Rate Interstital (MUPIT) Brachytherapy for Gynecologic Malignancies. Polish Journal of Medical Physics and Engineering, 16, 85-95.

https://doi.org/10.2478/v10013-010-0008-6

[14] Kandasamy, S., Reddy, K.S., Nagarajan, V., Vedasoundaram, P. and Karunanidhi, G. (2013) Dosimetric Impact of Inter-Fraction Variation in Interstitial HDR Brachytherapy. International Journal of Medical Physics, Clinical Engineering and Radiation Oncology, 2, 111-116. https://doi.org/10.4236/ijmpcero.2013.24015 
Submit or recommend next manuscript to SCIRP and we will provide best service for you:

Accepting pre-submission inquiries through Email, Facebook, LinkedIn, Twitter, etc. A wide selection of journals (inclusive of 9 subjects, more than 200 journals)

Providing 24-hour high-quality service

User-friendly online submission system

Fair and swift peer-review system

Efficient typesetting and proofreading procedure

Display of the result of downloads and visits, as well as the number of cited articles Maximum dissemination of your research work

Submit your manuscript at: http://papersubmission.scirp.org/

Or contact ijmpcero@scirp.org 